\title{
ENERGY TRANSFER PROCESSES IN HIGH-VOLTAGE CIRCUITS BASED ON MAGNETIC PULSE COMPRESSION
}

\author{
Oleksandr I. KHRYSTO \\ Department of Pulse Treatment of Disperse Systems \\ Institute of Pulse Processes and Technologies of NAS of Ukraine, Bogoyavlenskij avenue, 43-A, Mykolayiv, 54018, Ukraine, \\ Tel.+38 (0512) 55-82-52, E-mail: alexander.khristo@gmail.com
}

\begin{abstract}
This work focuses on analysis of different versions of modern high-voltage semiconductor-magnetic pulse generators (SMPG), which are an inalienable part of electric - discharge technologies that contain a serial or parallel converter stages in the input circuit. There is no in-depth study of the electrical, magnetic and energy characteristics of SMPG in well-known publications, since the processes corresponding to them in these circuits are considered only for a single cycle of SMPG operation and, in most cases, without determining the characteristics of saturable reactor (SR) magnetic flux density. The most widely used in practical applications are single-cycle SMPG, which in turn may differ in both the type of converter stages and their number. A special place among variety of $S M P G$ versions is allotted by single-switch and two-switch SMPG differing by serial, parallel or serial-parallel converter stage. The advantage of SMPG circuit with serial-parallel converter stage is to eliminate the use of additional bias source, because the magnetization reversal process of saturable reactor and pulse transformer (PT) takes place during the charging of storage capacitor of this stage. Each modification of pre-transformer compression stage has significant impact on the threshold of realization of asymmetric oscillation mode, especially for two - cycle operation SMPG circuits.
\end{abstract}

Keywords: saturable reactor, converter stage, semiconductor-magnetic pulse generator, asymmetric oscillation mode, magnetic compression stage, magnetization curve, numerical simulation

\section{INTRODUCTION}

Over the last decade or more an electrical discharge technologies for removal of hazardous pollutant in liquid and air environment have been noticeable developed [1-4]. An inalienable part of equipment of these technologies are high-voltage generator of short pulses, which are subjected to the special requirement for the parameters of the pulses being formed, such as peak power, pulse duration, leading edge and other. Most of these requirements can be satisfied by semiconductor-magnetic pulse generators (SMPG), which were significantly developed trough the emergency of new magnetic alloys with improved properties [5]. This achievement made it possible to reduce the dynamic switching losses of saturable reactors. Despite considerable progress in improving the technique of magnetic pulse generators are still problems with a reliable description of exchange processes occurring between the generator and load, their effect on the sustainable modes of oscillation and the efficiency of energy conversion in SMPG circuits.

\section{ANALYSIS OF THEORY AND PRACTICE OF SMPG APPLICATION}

The founders of the theory and development of magnetic generators are such scientists as L.A. Meerovich, I.S. Garber, G.F. Storm and M.A. Rosenblat. They laid the foundations of theory of steady-state oscillation mode of SMPG, carried out studies of the stability and region of an existence of this mode, proposed methods for calculating and constructing the compression stages of SMPG. Mainly for describing of energy transfer processes in charging circuit and first compression stage an idealized model of magnetization curve have been used, which is approximated by a broken line. According to this model, on the main magnetization curve a saturable reactor (SR) has infinitely large impedance, which gives reason to neglect magnetizing current. In the state of saturation of the core, the inductance of SR is determined from a saturation permeability, which is given by a constant independent of the magnetic field strength. However, the adopted idealization of the SR magnetization curve greatly restricts the studies of SMPG, which is especially important for understanding an energy transfer process in the absence of saturation SR delay between two successive stages of pulse compression. Furthermore, the SR model does not take into account the magnetizing currents and energy losses in the core due to the hysteretic nature of magnetization reversal. As a consequence the theory of the stationary oscillation mode being developed on the basis of idealized SR model is applicable only for the case of unidirectional energy transfer from generator to load and is a special case of whole variety energy modes of SMPG oscillation.

At the present time, a significant contribution to the development of scientific foundations, principles of construction and systematization of the SMPG was made by scientists of the Institute of Electrodynamics of the National Academy of Sciences of Ukraine, which developed a number of practical circuit diagrams of the SMPG with new pulse compression stages. These modern devices were used as high-voltage equipment for supplying a discharge chamber in the technology of water purification in a pulsed barrier discharge [6]. In particular, in the works of I.V. Volkova, V.I. Zozuleva, D.A. Sholokh [7] for the first time demonstrated the possibility of improving energy performance of the SMPG due to the simultaneous passage of currents in adjacent stages of pulse compression as well as circuit-node methods for increasing the energy and dynamic parameters of the SMPG by reducing core permeability coefficient of the SR in a saturated state.

Through to research aimed at finding more efficient electric discharge methods for cleaning air from industrial 
gas emissions, by scientists of IPPT of NAS of Ukraine have developed a special grade of high-voltage equipment that provides pulse shaping with a repetition rate of $10 \mathrm{kHz}$, a voltage up to $100 \mathrm{kV}$, a leading edge slope up to $1 \mu \mathrm{s}$, with pulse duration up to $20 \mu$ s. In particular, in the work of L.Z. Boguslavsky noted the positive effect of a dual high-voltage pulse on a gas purification efficiency during air treatment in streamer volumetric and corona discharges [8].

In present, one of the promising areas of nanosecond pulse technology is the use of a combination of an inductive storage and a semiconductor current interrupter in the terminate compression stage of SMPG circuit diagrams that allows to enhance the voltage amplitude and pulse power on the load. As semiconductor devices are used diodes with the effect of a sharp reverse current interruption $[9,10]$. The principle of operation of such circuits is realization of twocircuit pumping of diode structure with a regime of reverse current amplitude amplification, which is achieved by introducing a parallel stage of pulse compression between a serial compression stage and semiconductor diode.

The experience of modelling different versions of SMPG circuit diagrams and analysis of modern publications, including those shown above, does not show sufficient knowledge of the electrical, magnetic and energy characteristics of SMPG, which are mainly considered only for a single cycle of generator oscillations. Research in this area should contribute to the further improvement of these promising devices of converter technique.

\section{METHODS}

In this paper, we propose to use a comprehensive approach aimed at studying electromagnetic processes in high-voltage SMPG circuit diagrams, which takes into account the topology of the circuit, the design parameters of the saturable reactor and pulse transformer (PT), the magnetization curve, the equivalent load resistance, as well as temporal parameters of periodic commutating of power switches.

The goal of the work is to determine and compare the regularities of electromagnetic processes of energy transfer in high-voltage semiconductor - magnetic pulse generators with developed principles of constructing converter stages.

Formulation of the research problem: analyze the existing and develop new methods for constructing converter stage to improve the electrical and energy parameters of the SMPG; investigate the electromagnetic processes and steady-state modes of oscillations in highvoltage SMPG circuit diagrams with common topological principles of construction: a converter stage in the input circuit, an intermediate compression stage, a pulse transformer, a high-voltage compression stages.

\section{MAIN CONTENT OF WORK}

On the basis of numerical simulation and experimental research, an extended classification of controllable converter stages in the input circuit of SMPG was obtained, which separates these ones according to the number of controllable switches being used and the modification of the pre-transformer stage of magnetic pulse compression. Following this classification, it can be concluded that in single-switches circuits, the restoration of the initial state of the magnetic elements is achieved either by using a bias circuit or an inductive-diode charging circuit. However, these solutions have several disadvantages associated with a limitation of the pulse repetition rate and the discharge power. At the same time, the introduction of an additional element to the charging circuit allows separating charge and discharge conversion cycles, while the combination of a two-switch converter stage and a pre-transformer stage makes it possible to reveal new regularities in the functioning of structural stages and increase the energydynamic parameters of the generator.

Below are considered variants of high-voltage SMPG with various modifications of the converter and compression stages located in the primary circuit of the pulse transformer.

Taking into account the mentioned drawbacks of the SR model and in order to obtain a more approximate picture of the electromagnetic processes in the SMPG circuits, the SR model is taken as the basis for the magnetization characteristic, which is approximated by the arctangent function. The main provisions of the SR model are presented in [11]. The SMPG circuit diagram with a serial converter stage in the input circuit is shown in Figure 1. To implement the reverse recovery mode of the generator's elements, the SR $L_{1}$ and the PT $T_{\mathrm{p}}$ have corresponding additional windings $w_{12}, w_{23}$ being connected in series and being coupled to the same bias source $E_{\mathrm{b}}$ through the decoupling reactor $L_{\mathrm{d}}$. In order to analyze of electromagnetic processes a system of integral-differential equation with non-linear coefficient is compiled, which is

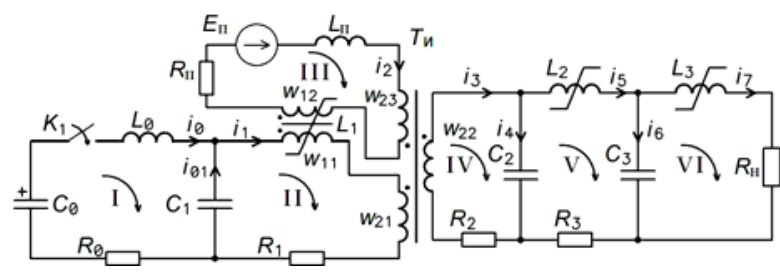

Fig. 1 SMPG circuit diagram with a serial converter stage in the input circuit and bias source.

solved numerically by the Euler method. The parameters of generator circuit elements and calculated system of equations are given in [12].

According to the simulation results, it is shown that the process of forming the working pulse is characterized by a gradual increase in the steepness of flux density in the SR cores and reduction of the SR saturation delay interval in the order of their arrangement in the generator circuit. At the same time, during the magnetization reversal time, the flux density in the SR $L_{1}$ changes according to a parabolic law, in $L_{2}$ close to parabolic, in $L_{3}$ it has the form of a rectangular pulse with damped oscillations on the shelf. The distinctive dynamics of flux density is explained by the fact that the magnetization reversal current of the SR $L_{1}$ and $L_{2}$ passes through the capacitor of the next compression stage, and the SR $L_{3}$ current through the linear load $R_{\mathrm{H}}$. The magnetization reversal process starts after the flux density of the first SR $L_{1}$ from the positive saturation threshold at a time of $25 \mu \mathrm{s}$ and ends after the input of the flux density of the final SR to the negative saturation threshold at a time of $90 \mu \mathrm{s}$. The rest of dynamics of the process is of the nature of damped oscillations and the longest turns out to be for 
the core of the SR $L_{3}$, in which the flux density approaches to the forced value only after $200 \mu$ s.

The superposed characteristics of flux densities in the SR cores and currents at the compression stages is shown in Fig. 2, which fully correspond to the theoretical basis on the magnetic generators, as well as were confirmed by experimentally in the work [13].

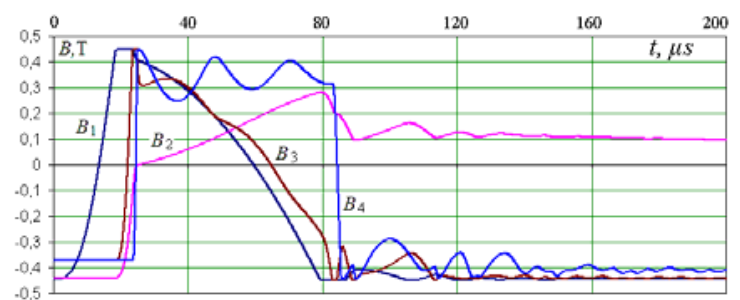

(a)

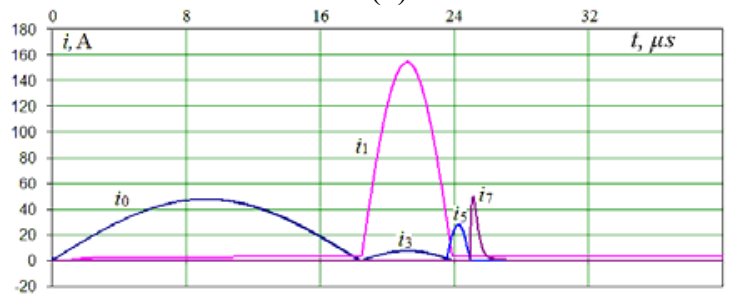

(b)

Fig. 2 Electrical and magnetic characteristics of the SMPG compression stages: a) dynamics of flux density in SR $L_{1}-L_{3}$ and transformer $T p ; b$ ) currents in the compression stages.

A distinctive feature has the characteristic of flux density $B_{2}$ in the core of the PT, for which the following main intervals can be distinguished: from $20 \mu \mathrm{s}$ to $25 \mu \mathrm{s}$ there is a sharp increase in flux density according to the parabolic law; from 25 to $140 \mu$ s, the flux density is characterized by a sequence of damped pulses of triangular shape; from $140 \mu \mathrm{s}$ and then the flux density decreases linearly. The increase in flux density over a time interval from $25 \mu \mathrm{s}$ to $80 \mu \mathrm{s}$ is explained by the fact that the bias current of the SR $L_{1}$ in its main winding has such a direction, at which the transformer core is magnetized towards the positive saturation threshold. It is clear that increasing the field strength in the magnetization winding of $L_{1}$ is impractical since it will only increase the magnitude of flux density in the transformer core. As the calculation shows, the same trend is observed with a decrease in the volume of the transformer magnetic circuit. At the moment of saturation of the SR $L_{1}$, a reverse polarity pulse is transmitted to the high-voltage capacitor $C_{2}$ but this pulse energy is not enough to return the flux density to original value at which the SR $L_{1}$ leaves the positive saturation threshold. Starting from $140 \mu$ s, the flux density is linearly reduced only due to the total action of the bias currents.

In the other hand, simulation shows that during the charge of the capacitor $C_{1}$ due to the mutual coupling between the windings of the SR $L_{1}$, a part of the energy of the working pulse will be transferred to the bias source $E_{\mathrm{b}}$ which will lead to the appearance of a current pulse in the circuit of this source. At the flux density recovery stage, the accumulated energy is retuned back to the capacitor of the compression stage. However the energy recovery process breaks down after saturation of the SR $L_{1}$, which will affect on the subsequent dynamic of flux density in the PT $T_{\mathrm{P}}$. In addition, the current in the bias circuit takes a value higher than the original one. This circumstance may disrupt the condition for the existence of a SMPG stationary oscillation mode.

The calculations show that the flux density characteristic in the PT core substantially depends on the magnitude of the inductance of the decoupling reactor and on the mutual induction coefficient between the windings of the SR $L_{1}$. Figure 3 shows the superposed characteristics of flux density in the PT and currents in the bias source for the three values of the decoupling inductance: $1-100 \mu \mathrm{H}$, $2-200 \mu \mathrm{H}, 3-320 \mu \mathrm{H}$ and with the maximum coupling coefficient between the windings of the SR $L_{1}$.

It is found that a decrease in the current increment in the bias source allows improving the dynamics of $B_{2}$ in PT, and as a result to reduce the time its magnetization reversal. It is shown that if the coupling coefficient between the SR

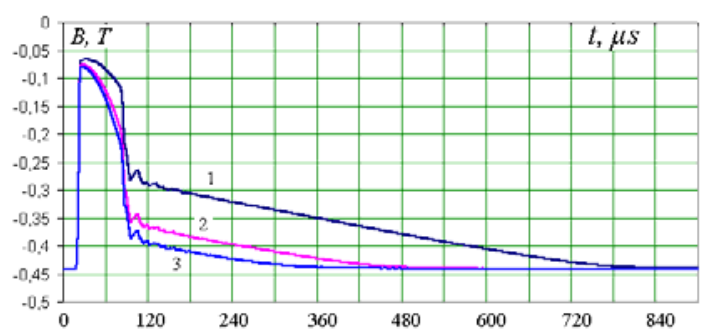

(a)

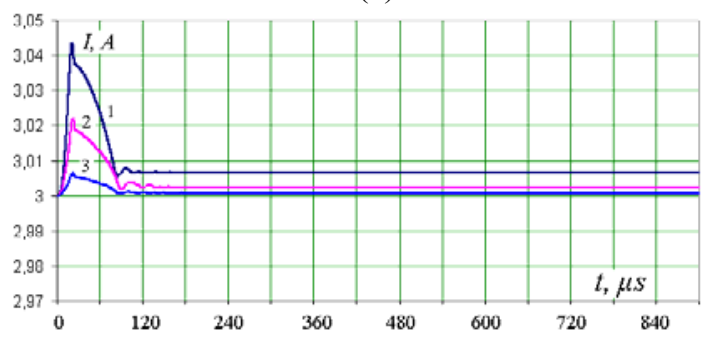

(b)

Fig. 3 Superposed characteristics of: a) flux density in the PT; b) current in the bias source.

windings $L_{1}$ is weakened and the inductance of the decoupling reactor increases, it is possible to significantly reduce the PT magnetization reversal time. When fulfilling the requirements to reduce the current increment in the bias source circuit, the frequency of SMPG operation can be increased to $5 \mathrm{kHz}$ or more.

In the designs of modern SMPG, there is a tendency to exception the bias source due to the influence of the magnitude of the bias current on the energy of the reverse pulse. Increasing the reversed polarity pulse energy is undesirable for some electrical discharge loads. For example, in the power supply sources of copper bromide vapour lasers, a charge bypass reactor connected in parallel to the laser tube is used to eliminate the undesired reverse polarity pulse. An alternative replacement for SMPG with bias current is SMPG with a two-switch converter in the input circuit. The electrical circuit of this version of SMPG is shown in Fig.4.

The main advantage of the circuits is that the magnetization recover process is carried out simultaneously with charging of the longitudinal capacitor $C_{1}$ of the converter stage.

Modelling of SMPG shows that in the circuit diagram, in addition to the asymmetric regime of oscillation there is self-excitation regime, which is realized, when the integral value of the discharge voltage across the winding of the SR exceeds the permissible flux density swing in the SR core 


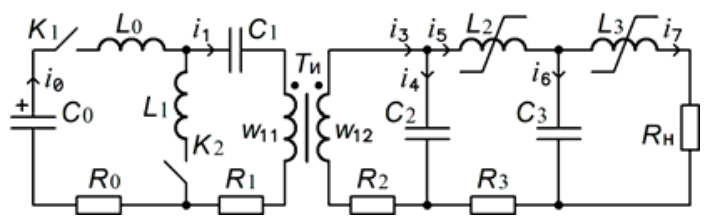

Fig. 4 SMPG circuit diagram with a serial-parallel stage in the input circuit.

under the condition that the charging current exceeds the duration of the discharging current. The transient process of self-excitation of SMPG is characterized by a gradual increase in the amplitude of oscillations on the longitudinal capacitor of the converter stage, as well as an increase in the amplitude and energy of the inverse pulse on the load. The parameters of computational model of the generator circuit and the finite-difference recording of the system of integral-differential equations are given in [14]. Figure 5 shows the electromagnetic characteristics in the SMPG of the transient process of the asymmetric mode with a free discharge of the capacitor $C_{0}$. In the transient regime, the forced oscillations that lead to an increase in the amplitude of the voltage across the capacitor $C_{1}$ are excited.

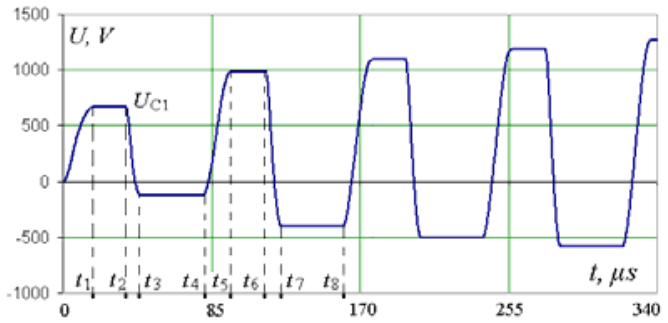

(a)

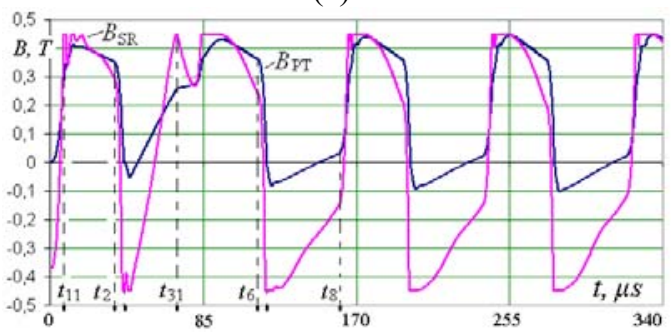

(b)

Fig. 5 Electromagnetic characteristics at the initial stage of transient process in SMPG: a) voltage across $C_{1} ; \mathrm{b}$ ) superposed characteristics of flux density at PT and SR $L_{2}$

In the time interval $0-t_{1}$, through the open switch $K_{1}$ occurs the charge of the longitudinal capacitor $C_{1}$ and the high-voltage $C_{2}$. A sufficiently long period of charging current introduces high-voltage SR $L_{2}$ into a state of positive saturation, creating an oscillatory circuit $C_{2}-L_{2}-C_{3}$ that limits the amplitude of the forward pulse and the energy transfer to the load. At time $t_{1}$, the charging current drops to zero and the energy in the longitudinal capacitor reaches its maximum, which is higher than for the symmetric mode of oscillations. At the time $t_{2}$, the switch $K_{2}$ is opened and $C_{1}$ is discharged to $C_{2}$, and an inverse voltage pulse with amplitude of the order of $15 \mathrm{kV}$ is formed on the latter. Since the SR $L_{2}$ is not designed for the stored energy in $C_{1}$, the process of charging $C_{2}$ terminates as a result of achieving of flux density in the SR $L_{2}$ of the negative saturation threshold. At time $t_{3}$, the discharge current through the switch $K_{2}$ drops to zero. As a result of mismatched energy transfer, on the capacitors $C_{1}$ and $C_{2}$ there will appear residual voltages of different polarities. Under the influence of positive residual voltage on $C_{2}$, flux density in SR $L_{2}\left(B_{2}\right)$ will reach a positive saturation threshold, before switching $K_{1}$. At time $t_{4}$, the cycle is repeated and the switch $K_{1}$ is opened, due to the negative residual voltage on $C_{1}$, the voltage charging level by the time $t_{5}$ becomes even greater than at time $t_{1}$. Since the flux density in the SR $L_{2}$ and the transformer is near to the positive saturation threshold, then on $C_{2}$ there appears a non-significant voltage pulse. At the time $t_{6}$, the switch $K_{2}$ is reopened, the discharge current increases and an inverse pulse of even greater amplitude is formed on $C_{2}$ than the previous one. Thus, due to incomplete energy transfer, the system operates with a positive feedback, leading to an amplification of the input signal. It is important to note that in the interval $t_{7}-t_{8}$ due to a small residual voltage on $C_{2}$, the $L_{2}$ and PT will be partially magnetized to a positive saturation threshold. At time $t_{8}$, the flux density swing in PT and SR $L_{2}$ will be significantly larger than the time $t_{4}$, and the charging voltage for $C_{1}$ will increase. In the subsequent switching of the $K_{1}$, the level of the residual voltage on $C_{2}$ will increase, and the flux density swing will decrease to the moment of commutation $K_{2}$.

It turns out that the transient process of the selfexcitation regime in the two-switch SMPG is aimed at levelling the paths of the charging and discharging currents. The transient process will adopt a steady-state level of amplitude oscillation when a significant portion of the discharge current passes successively in the winding of all high-voltage SR and closes on the load. In addition, an increase in the input current and voltage leads to a shift of flux density in the PT to a negative saturation threshold. At the end of the transition process, the lower level of flux density in the PT will reach a negative saturation threshold, which will limit the amplitude of the inverse pulse on the high-voltage capacitor. For this computational model, the transient oscillation mode will end after $4 \mathrm{~ms}$. If the energy of the power supply capacitor is kept unchanged, the voltage amplitude on the longitudinal capacitor $C_{1}$ increases from $500 \mathrm{~V}$ to $2 \mathrm{kV}$, and the amplitude of the forward and inverse pulses in $C_{2}$ are 5 and $20 \mathrm{kV}$, respectively.

Fig. 6 shows the electrical and power characteristics of the parallel converter stage, depending on the equivalent load resistance in the range from 60 to $300 \mathrm{Ohm}$ and for three values of the supply voltage $-1-350 \mathrm{~V}, 2-400 \mathrm{~V}, 3$ - $480 \mathrm{~V}$. The family of peak current and voltage characteristics through the converter element $K_{2}$, stored energy in the capacitor $C_{1}$ have a similar character that can be approximated closely by a hyperbolic function. On each characteristic, it is possible to distinguish a section with a linear dependence and a region where the characteristic has a high steepness of growth. In the range of equivalent load resistances from 100 to $300 \Omega$ at $350 \mathrm{~V}$ of input power, the parallel converter stage has the most linear characteristics that are practically independent of the load resistance. With an increase in the supply voltage, the region where the characteristics of the converter stage are linear in nature is narrowed towards higher load resistances. It is shown that the linearity of the characteristics of the converter stage in the appropriate range of load resistance at a certain value of the input voltage is explained by the coordinated energy transfer from the low-voltage capacitor to the high-voltage 


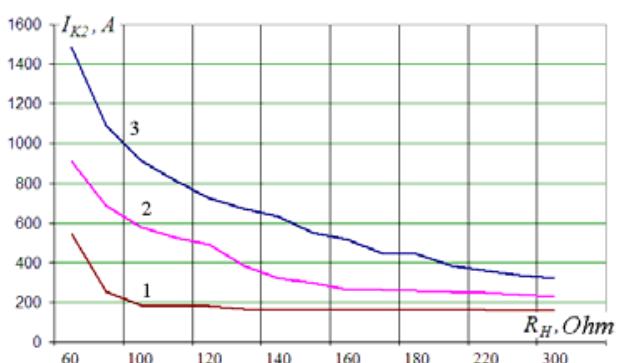

(a)

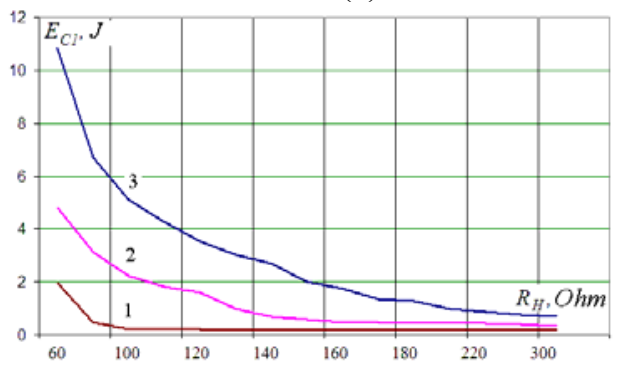

(b)

Fig. 6 Family of electrical and energy characteristics of parallel converter stage, depending on the equivalent load resistance: a) peak current through the switch $K_{2}$; b) stored energy of the parallel converter stage.

capacitor. So, at a supply voltage of $350 \mathrm{~V}$ and in the range of equivalent load resistances from 100 to $300 \mathrm{Ohm}$, the greatest efficiency of the converter stage is achieved, which lies in the range from 0.8 to 0.9 .

It is found that the nature of the course of electromagnetic processes in the circuits of the two-switch SMPG is significantly influenced by the presence of a pretransformer stage of magnetic compression. The possible variants of magnetic compression stages in the primary PT circuit are shown in Fig. 7. According to the obtained classification it is possible to distinguish two-switch converter stages containing serial (Fig. 7, a), serial-inverse (Fig. 7, b) and a recharge-serial (Fig. 7, c) pre-transformer stage of magnetic compression. As simulation shows, the introduction of the magnetic compression stage increases the threshold for the existence of an asymmetric mode of oscillation in the SMPG. However, this solution reduces the load on power switches and optimizes the duration of current pulses through PT.

So for the circuit diagram with a serial compression stage (Fig. 7, a), it is found that in the range of the input supply voltage up to $350 \mathrm{~V}$ in the generator only symmetrical mode of oscillations is observed. The parameters of this generator circuit diagram and the details of the computational model are given in [15]. When the capacitance of $C_{1}$ is doubled in relation to $C_{2}$ capacitance, the threshold voltage of the asymmetric mode can be reduced to $150 \mathrm{~V}$. Also for this circuit, it is noted that the transverse capacitor $C_{2}$ has virtually no effect on the charge of the longitudinal capacitor $C_{1}$ due to the charging current flowing through the saturated SR and the primary winding PT. The nature of the change in currents and voltages in the converter stage and the pre-transformer compression stage is shown in Fig. 8, where $U_{\mathrm{C} 2}, U_{\mathrm{C} 2}$ are the voltages on the capacitors $C_{1}$ and $C_{2}$, respectively, $i_{0}$ and $i_{1}$ are the currents through the converter and compression stages, respectively. A preliminary positive saturation of the high-voltage SR creates an oscillatory circuit that acts as a low-pass filter, which prevents further energy transfer to the load in the process of charging the capacitor $C_{1}$. Due to the increased energy of the power source necessary to realize the asymmetric mode of oscillations, the SR $L_{2}$ will be saturated before the complete energy transfer from $C_{1}$ to $C_{2}$ occurs and the discharge current will become protracted. Under the influence of a negative residual voltage on $C_{2}$, the $L_{2}$ SR $L_{2}$ will be reversal magnetized to the positive saturation threshold (the initial state) before the commutation of the switch $K_{2}$.

Simulation shows that the stationary mode of asymmetric oscillations for this circuit, provided that the capacitances $C_{1}$ and $C_{2}$ are equal, is realized when the output load is in the range from 70 to $110 \mathrm{Ohm}$, that is $45 . . .80 \%$ of the matched load.

Fig. 9 shows the electrical and power characteristics of

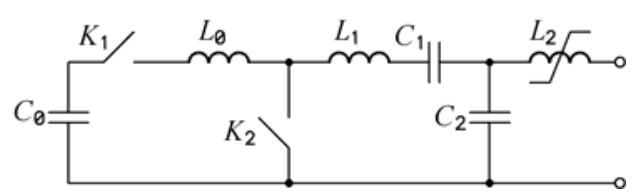

(a)

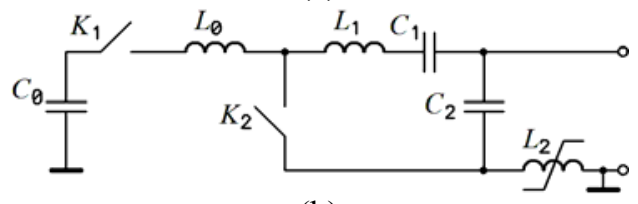

(b)

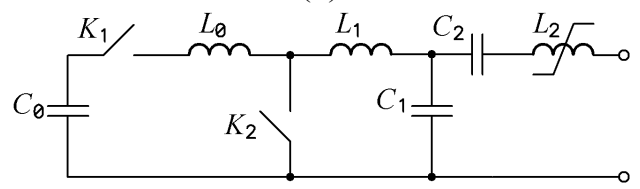

(c)

Fig. 7 Variants of converter stages in the input circuit of SMPG.

the parallel converter stage, depending on the supply voltage for two values of the equivalent load resistance - 1 - $80 \mathrm{Ohm}, 2$ - $120 \mathrm{Ohm}$. In contrast to the previous circuit diagram, it was found that the electrical and energy characteristics of the parallel converter (Fig. 9) become more linear and have much smaller angular coefficient. It

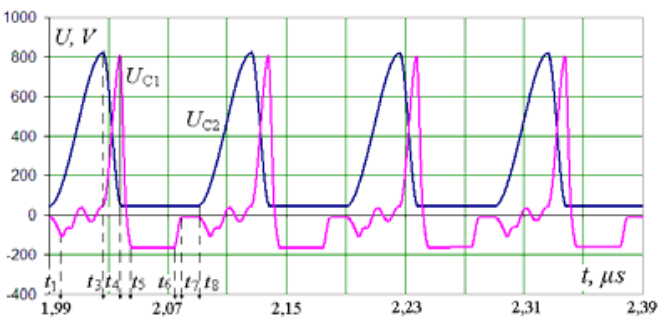

(a)

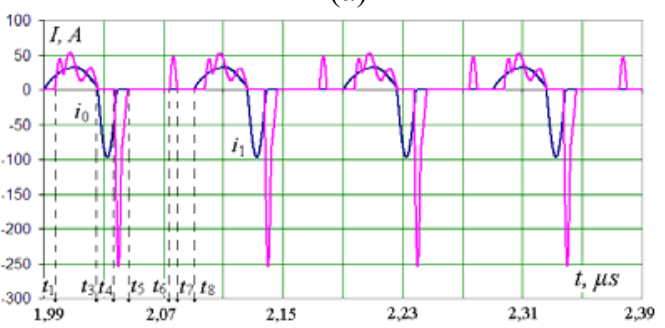

(b)

Fig. 8 Electrical characteristics of the serial converter stage of SMPG: a) superposed characteristics of currents trough $C_{1}$ $-i_{0}$ and trough primary circuit of PT $-i_{1} ; \mathrm{b}$ ) superposed characteristics of voltages on $C_{1}$ and $C_{2}$. 


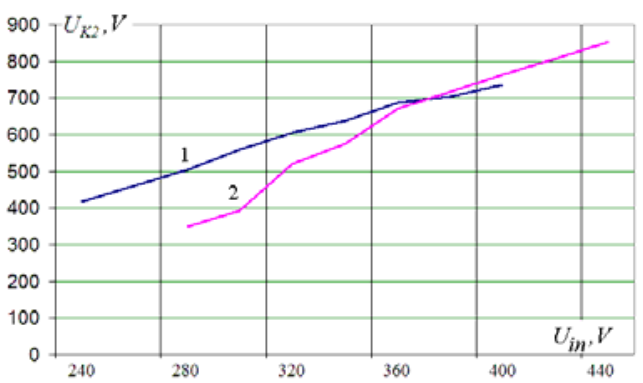

(a)

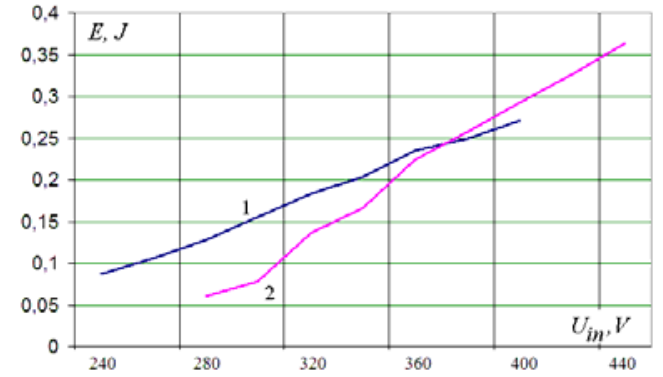

(b)

Fig. 9 Electrical and energy characteristics of the parallel converter stage, depending on the supply voltage: a) peak voltage across the switch $K_{2}$; b) stored energy in the capacitor $C_{1}$.

is also found that the noted solution allows maintaining a higher value of the efficiency of the converter stage in a wide range of the supply voltage. By running the model, it is possible to adjust the inductance of the SR $L_{2}$ (the number of turns, the cross section of the magnetic core) so as to maximally reduce the amplitude of the forward pulse and improve the single-polarization effect. Therefore, the introduction of an additional compression stage, as done in [16], is justified, most likely, by improving the conditions for matching the compression stage with PT.

A distinctive feature of the circuit with a serial - inverse pre-transformer compression stage (Fig. 7, b) is that the charge current of the capacitor of the converter stage passes through the circuit covered by the primary circuit bypassing SR $L_{2}$. However, still part of the charging current will branch along the $C_{1}-L_{1}$ circuit, slightly magnetizing the SR $L_{2}$ to a negative saturation threshold. The electrical characteristics of the converter are shown in Fig. 10, where $i_{0}$ is current through the $C_{1}, i_{1}$ is current through primary circuit of PT, $U_{\mathrm{C} 2}$ and $U_{\mathrm{C} 2}$ are the voltages on the capacitors $C_{1}$ and $C_{2}$, respectively. The designations of currents and voltages correspond to the characteristics by analogy as in the previous circuit diagram. Due to the fact that the SR $L_{2}$ on the main magnetization curve has a large inductive resistance, then on the charging interval the transverse capacitor remains practically discharged. When an inverse pulse is formed, the current through the converter element will take a trapezoidal character (Fig. 10), since after saturation of the SR, the energy will be introduced simultaneously into two capacitors. It is also found that the discharge current of the low-voltage capacitor will be superimposed on the discharge currents of the high-voltage capacitors in a sequential order according to the stage of saturation of the SR. The resulting current through the load will be the total discharge current of all four capacitors. This effect makes it possible to increase the energy transferred to the load by an order of magnitude in comparison with the existing mode of oscillations. The

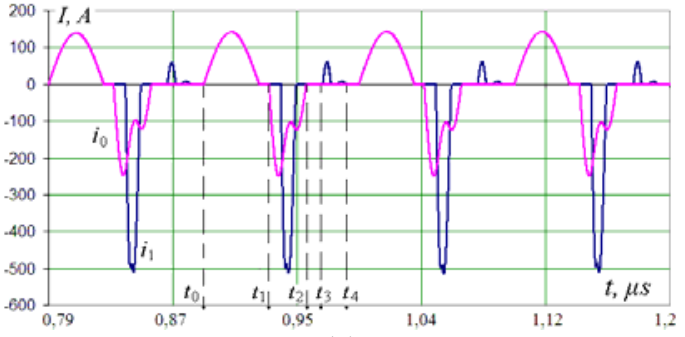

(a)

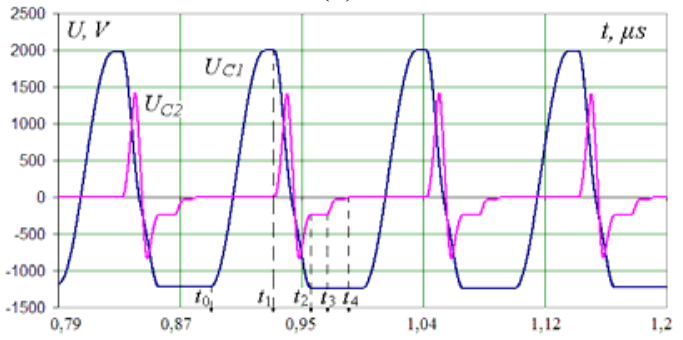

(b)

Fig. 10 Electrical characteristics of the serial - inverse converter stage of SMPG: a) superposed characteristics of currents $i_{0}$ and $i_{1} ; \mathrm{b}$ ) superposed characteristics of voltages on $C_{1}$ and $C_{2}$.

main drawback of the serial-inverse SMPG circuit diagram is that the SR operates on a partial hysteresis loop, which requires an increase in its size for the realization of the stationary mode of oscillations.

The circuit diagram with a recharge-serial compression stage (Fig. 7, c) is a reconfiguration of the previous circuit in which the transverse and longitudinal capacitors are rearranged in place. This circuit solution allows the pulse voltage in the primary PT circuit to be increased by recharging the transverse capacitor $C_{1}$ through the parallel converter element $K_{2}, L_{1}$. As a result of the conversion, the energy from two successive capacitors $C_{1}$ and $C_{2}$ is applied to the SR $L_{2}$, which causes a rapid magnetization $L_{2}$ to a negative saturation threshold. After saturation of $L_{2}$, the energy from $C_{1}$ and $C_{2}$ is transferred to the high-voltage capacitor, however, since the discharge is not perfectly matched, then at the end of the discharge process, negative residual voltages will appear on the capacitors $C_{1}$ and $C_{2}$. Under the action of these voltages, the SR $L_{2}$ is remagnetized to a positive saturation threshold that during the formation of the charging pulse it will provide an unobstructed path of flow of the current of the longitudinal capacitor and accordingly the asymmetry of the pulses.

On the basis of a comparative analysis of the electrodynamic characteristics of the SMPG being considered, it is found that the SMPG with a recharge-serial compression stage in contrast to other circuit diagrams has a noticeable advantage over the peak and average power that is released in the load, even though this SMPG version has a lower boundary pulse repetition rate. The increase in the peak power is due to the fact that in the recharge-serial compression stage, energy is stored not only in the longitudinal capacitor, as in other SMPG, but also in the transverse capacitor, which is recharged in the next cycle. In addition, in order to achieve a minimum threshold for the existence of an asymmetric mode of oscillation, in this case a significantly lower value of the input supply voltage is required, rather than for circuit Fig. 7, a. The dynamics of the voltages in the SMPG compression stages are shown in Fig. 11. 


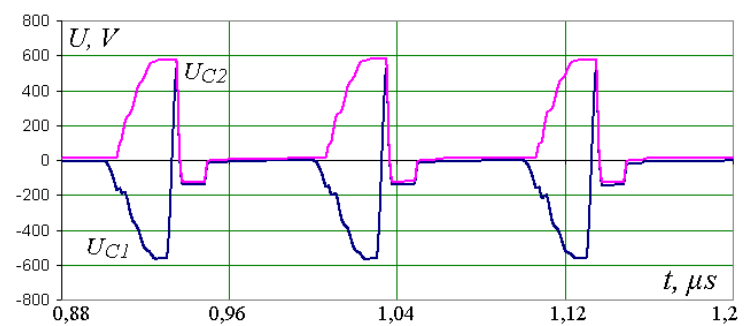

(a)

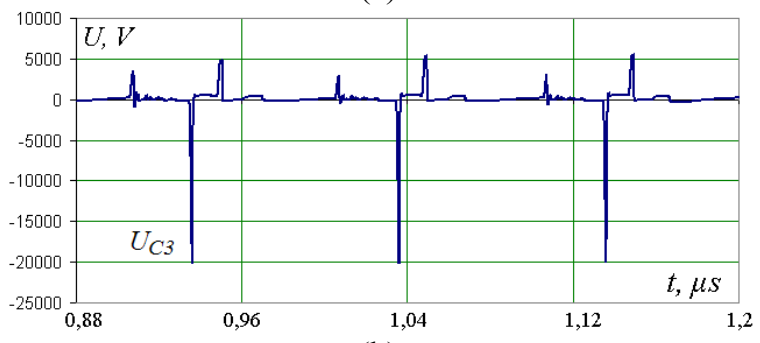

(b)

Fig. 11 Electrical characteristics of stationary mode of asymmetric oscillations of SMPG: a) superposed characteristics of voltages on $C_{1}$ and $C_{2} ; \mathrm{b}$ ) characteristic of voltage on $C_{3}$.

Subject to the optimal energy balance between the capacitors $C_{1}, C_{2}$ and $C_{3}$ (high voltage capacitor), the pulse duration can be reduced by half, which will make it possible to generate pulses with a higher peak power on the load than in the previous circuit diagram.

Regulation of the amplitude of the output pulses of SMPG in the above versions of the converter stages is carried out by changing the voltage level of the DC power supply. However, this is not the only method for controlling the amplitude of pulses. There is another option to control the amplitude of the output pulses of SMPG [17], which is achieved by controlling the duration of the charge or discharge currents of converter stage at an unchangeable input voltage of the DC power supply. The complexity of implementing proposed method of pulse amplitude controlling is in suppressing voltage spikes across controlled switches caused by an abrupt change in the direction of current flow through inductive circuit elements, which requires using an additional means to return unspent energy back to the storage capacitors of the power supply or converter stage.

\section{CONCLUSIONS}

1. A classification of controllable converter stages in the input circuit of SMPG is proposed, which differs from the known one in that it separates these stages depending on the number of semiconductor switches being used and the modification of pre-transformer compression stage.

2. In the SMPG circuit diagram with magnetization of its elements, the longest dynamics of magnetization reversal is observed in the core of a pulse transformer. Perfect conditions of magnetization reversal of PT can be achieved by increasing the ratio of the inductance of decoupling reactor to the inductance of charge reactor, or by weakening the coupling coefficient between the windings of the SR $L_{1}$. 3 . In the SMPG circuit diagram with a parallel converter in the input circuit, compliance with the conditions of the inequality of charge and discharge inductances $\left(L_{0}>L_{1}\right)$ and excess of the limiting energy switched by high-voltage SR leads to the development of self-excitation process, which is characterized by a gradual increase in voltage on the longitudinal capacitor, an increase in the amplitude of inverse pulse and its energy on the load.

4. The increase in voltage on the longitudinal capacitor occurs due to the appearance of a negative residual voltage on it as result of incomplete transfer of energy from this capacitor to the high-voltage capacitor during the formation of the inverse pulse. In the subsequent charge cycle, the level of charging voltage on the longitudinal capacitor becomes larger by the amount of residual voltage from a previous conversion step.

5. The addition of serial pre-transformer stage for the pulse compression in the two-switch SMPG circuit diagram makes it possible to improve the conditions of magnetization reversal of compression stage, reduce the load on the semiconductor switch, optimize the pulse width in the input of PT and linearize the dependence of the peak values of electrical characteristics of the converter stage on the supply voltage level.

6. The SMPG circuit diagram with a recharge-serial compression stage in contrast to the SMPG circuit diagrams with other modifications of pre-transformer compression stages, taking into account the same values of the input voltage and equivalent load resistance has a shortest duration of the output pulse at its largest amplitude, which makes it possible to obtain the highest peak and average power on the load.

\section{ACKNOWLEDGMENTS}

I am immensely grateful to the staff of the Institute of Electrodynamics of NAS of Ukraine, especially V. I. Zozulev, whose inventive activity and peculiar comprehension of the finest perfecting of power converters were of paramount importance in the further development of the technology of semiconductor - magnetic pulse generators.

\section{REFERENCES}

[1] POKRYVAILO, A. - YANKELEVICH, Y. - WOLF, M.: A High-Power Pulsed Corona Source for Pollution Control Applications, IEEE Transactions on Plasma Science, Vol. 32, No. 5, pp. 2045-2054, 2004. https://doi.org/10.1109/tps.2004.835952.

[2] SHENGLI, LI. - SHENG, HU. - HAN, Z.: A Novel Nanosecond Pulsed Power Unit for the Formation of $\mathrm{OH}$ in Water, Plasma Science and Technology, Vol. 14, No. 4, pp. 312-315, 2012. https://doi.org/10.1088/1009-0630/14/4/08

[3] PEREVERZEV, A. V. - LITVINENKO, T. M.: Highvoltage converter for electrodischarge neutralization of sulfur dioxide, Tekhnichna Elektrodynamika, No. 6, pp. 84-89, 2015. http://techned.org.ua/2015_6/st15.pdf

[4] JIANG, W. - YATSUI, K. - TAKAYAMA, K.: Compact solid-state switched pulsed power and its applications, Proceedings of the IEEE, Vol. 92, No. 7, pp.1181-1196, 2004.

https://doi.org/10.1109/JPROC.2004.829003 
[5] CHOI, J.: Introduction of the magnetic pulse compressor (mpc) - fundamental review and practical application, Journal of electrical engineering and technology, Vol. 5, No. 3, pp. 484-492, 2010.

https://doi.org/10.5370/JEET.2010.5.3.484

[6] BOZHKO, I. V. - ZOZULEV, V. I. KOBYLCHAK, V. V.: SOS - generator for electrodischarge technologies used pulse barrier discharge, Tekhnichna Elektrodynamika, No. 2, pp. 63-67, 2016.

https://doi.org/10.15407/techned2016.02.063

[7] VOLKOV, I. V. - ZOZULEV, V. I. - SHOLOKH, D. O.: Foundations of constructing compression magnetic semiconductor devices for both electropulse technology and pulsed lasers, Tekhnichna Elektrodynamika, No. 3, pp. 10-18, 2011. http://techned.org.ua/article/11-3/st2.pdf

[8] VOVCHENKO, A. I. - BOGUSLAVSKY, L. Z. MIROSHNICHENKO, L. N.: Trends in the development of high-power high-voltage pulse current generators at the IIPT of the National Academy of Sciences of Ukraine, Tekhnichna elektrodynamika, No 5. pp. 69-74, 2010.

[9] RUKIN, S.N.: Pulsed power technology based on semiconductor opening switches: A review, Instruments and Experimental Techniques, Vol. 91, No. 1, 2020.

https://doi.org/10.1063/1.5128297

[10] HU, L. - DING, Z. - HAO, Q.: Design of a $43 \mathrm{kV}$, $20 \mathrm{kHz}$ solid-state pulse generator driven by a lowcurrent density-pumped semiconductor opening switch, Measurement Science and Technology, Vol. 24, No. 7, p. 4, 2013. https://doi.org/10.1088/0957$0233 / 24 / 7 / 077002$

[11] KHRYSTO, O. I. - ZOZULEV, V. I. - SHOLOKH, D. O.: Numerical modeling of electromagnetic processes in the circuit diagram of a magnetic pulse generator, Tekhnichna Elektrodynamika, No. 2, pp. 22-28, 2014.

http://techned.org.ua/2014_2/st4.pdf

[12] ZOZULEV, V. I. - SHOLOKH, D. O. - KHRYSTO, O. I.: Simulation of electromagnetic processes in the circuit of semiconductor - magnetic direct pulse generator, Pratsi Instytutu Electrodynamiky NAN Ukrainy, No. 3, pp. 15-24, 2015.

[13] ZOZULEV, V. I. - SHOLOKH, D. O. KOBYLCHAK, V. V. - KHRYSTO, O. I.: The main trends of semiconductor magnetic-pulse generators, Tekhnichna Elektrodynamika, No. 4, pp. 47-49, 2016. https://doi.org/10.15407/techned2016.04.047

[14] KHRYSTO, O. I.: Electrical and energy characteristics of the serial-parallel converter unit of a high-voltage semiconductor - magnetic pulse generator, The Bulletin of National Technical University "KhPI". Series: Techniques and Electrophysics of High Voltage, No. 51, pp. 99-106, 2015.

[15] VOLKOV, I. V. - ZOZULEV, V. I. - KUSKOVA, N. I. - KHRYSTO, O. I.: Development of principles for constructing a pre-transformer part of magneticsemiconductor pulse generators, Pratsi Instytutu Electrodynamiky NAN Ukrainy, No. 47, pp. 45-53, 2017. https://doi.org/10.15407/publishing2017.47.045

[16] ZOZULEV, V. I. - SHOLOKH, D. O. KOBYLCHAK, V. V. - KHRYSTO, O. I.: New aspects of magnetic-pulse semiconductor devices improvement, In: Intelligent Energy and Power Systems. Kyiv: IEPS, pp.209-214, 2016. https://doi.org/10.1109/IEPS.2016.7521870

[17] VOLKOV, I. V. - ZOZULEV, V. I. - KHRYSTO, O. I.: Increasing of the efficiency of power electronics devices by the control of charging time of the capacitors in their circuits, Tekhnichna Elektrodynamika, No. 2, pp. 15-18, 2019. https://doi.org/10.15407/techned2019.02.015

Received February 27, 2020 , accepted July 10, 2020

\section{BIOGRAPHY}

Oleksandr Khrysto was born in Mykolaiv, Ukraine, in July 1985. He began his career at the Institute of Pulse Processes and Technologies of the National Academy of Sciences of Ukraine as an engineer since April 1, 2008 after obtaining the specialist degree in electronics engineering from Admiral Makarov National University of Shipbuilding in the specialty "Electronic Systems". During the period from 2009 to 2012 he studied in graduate school of Institute of Pulse Processes and Technologies (IPPT) of the NAS of Ukraine on specialty 05.03.07 - "Processes of Physical and Technical Processing" (scientific director doctor of technical sciences, professor, Kuskova N.I.). He defended his degree of candidate of technical sciences by specialty 05.09.12 - power semiconductor converters in 2017; his thesis title was "High-voltage generators of submicrosecond pulses with improved energydynamic parameters of each unit for electric discharge technologies". Since 2019 he has been working as senior lecturer at Petro Mohyla Black Sea National University at the Faculty of Computer Science. His research interests: high-voltage magnetic pulse generators, numerical simulation, electronic systems, semiconductor power converters, electrical discharge in condensed matter. 